Prophylaktische Nitroglycerin-Gabe bei KHK-Patienten \title{
Training ohne Angst und Schmerzen
}

\section{Etwa ein Drittel der Patienten mit chronischer KHK haben auch nach einer interventionellen Therapie weiterhin Angina-pectoris-Be- schwerden. Eine Trainingstherapie mit gezieltem Einsatz kurz wirk- samer Nitrate (z. B. Nitrolingual ${ }^{\oplus}$ akut Spray) kann die Belastbarkeit dieser Patienten erhöhen.}

- Trotz des erheblichen therapeutischen Aufwands einschließlich einer prophylaktischen Medikation mit ASS, Betablocker, ACE-Hemmer/Sartan und Statin leiden viele Patienten weiterhin unter pectanginösen Beschwerden, so Prof. Christian Holubarsch, Bad Krozingen. In einer Studie wurde gezeigt, dass fünf Jahre nach erfolgter $\mathrm{PCl}$ trotz optimaler medizinischer Behandlung mehr als 25\% der Patienten weiterhin Angina-pectoris-Anfälle haben.

Die Ursachen sind vielfältig. Betroffen sind Patienten, die Koronarinterventionen und Herzchirurgie verweigern oder aufgrund der Ko- und Multimorbidität für diese Eingriffe nicht infrage kommen. Auch inkomplette oder frustrane Rekanalisierung oder ein Bypassverschluss können Gründe sein. Häufig findet man aber auch Angina-pectoris-Anfälle nach erfolgreicher PTCA und Stent-Implantation. Hier liegt dann häufig eine endotheliale Dysfunktion zugrunde, meinte Holubarsch.

Die positiven Auswirkungen einer medizinischen Trainingstherapie bei stabiler KHK sind vielfach belegt. Dabei wurde beobachtet, dass sich die Angina-pectorisSchwelle durch die prophylaktische Anwendung eines Nitroglycerin-Sprays vor der Belastung anheben lässt. Dies hat zur Folge, dass die Patienten länger ohne Ischämie trainieren können, was das Training effektiver und angstfreier werden lässt, sagte der Kardiologe.

Dem Effekt der prophylaktischen Nitroglycerin-Gabe möchte man jetzt im Rahmen einer placebokontrollierten Doppelblindstudie nachgehen. Bei insgesamt 20 KHK-Patienten wird untersucht, welchen Einfluss die Gabe von Nitroglycerinspray unmittelbar vor der Belastung auf die Zeitdauer bis zum Erreichen der Angina-pectoris-Schwelle oder bis zum Auftreten von ST-Streckensenkungen hat.

\section{Paradoxe Vasokonstriktion verhindern}

Auch Prof. Rainer Hambrecht, Bremen, unterstrich den hohen Stellenwert der Akutnitrate bei der Therapie der stabilen KHK mit Angina pectoris. Durch die häufig vorliegende endotheliale Dysfunktion mit NOMangel kommt es unter Belastung nicht sel-
Abbildung 1

Erhöhung der Belastungsdauer in Minuten

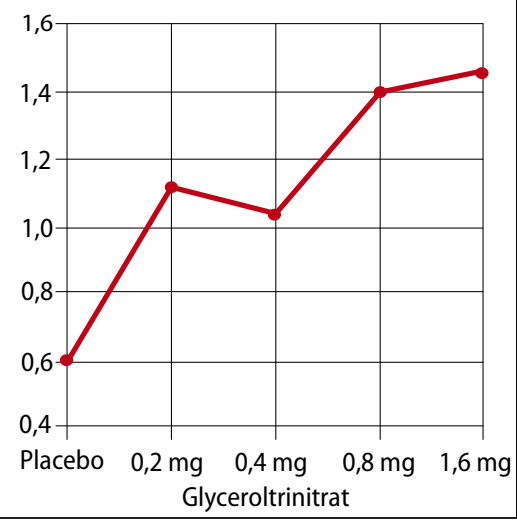

Ein kurz wirksames Nitrat erhöht die Belastbarkeit bei KHK-Patienten.

ten zu einer paradoxen Vasokonstriktion, die durch die prophylaktische Gabe von $\mathrm{Ni}$ troglycerin effektiv verhindert werden kann. Die Angina-Schwelle wird angehoben und die maximale Belastungsdauer nimmt zu. Auch in der Leitlinie der europäischen Gesellschaft für Kardiologie wird Nitro-Spray neben der Anwendung zur Anfallskupierung - prophylaktisch vor Belastungssituationen empfohlen. Hambrecht empfahl, allen KHK-Patienten ein Nitro-Spray zu verordnen und sie über den korrekten Gebrauch zu informieren.

\section{- Maria Weiß}

Quelle: Satellitensymposium auf der 38. Jahrestagung der Deutsch. Gesell. für Prävention und Rehabilitation von Herz-Kreislauferkrankungen, Juni 2011, Berlin (unterstützt von Pohl Boskamp)

\section{Nach kardioembolischem Schlaganfall}

\section{Auch bei Betagten: keine Scheu vor der Antikoagulation}

— Vorhofflimmern ist ein Risikofaktor für den Schlaganfall und damit auch für die Entwicklung einer Demenz. Problematisch ist laut Prof. Martin Grond, Siegen, dass viele kleinere kardioembolische Insulte nicht diagnostiziert werden. Aber auch wenn der Hirninfarkt bekannt ist, erfolgt oftmals keine adäquate Sekundärprävention. Gründe sind vor allem das Alter der Patienten, Gebrechlichkeit, eine erhöhte Sturzgefahr, eine bekannte Multimorbidität und vermutete Complianceprobleme.

Dass eine effektive und sichere Antikoagulation auch bei diesen Patienten möglich ist, zeigte eine Subgruppenanalyse der RELY-Studie, in der 18113 Vorhofflimmern-Patienten mit dem oralen direkten Thrombininhibitor Dabigatran (Pradaxa ${ }^{\circledR}$ ) oder dem Vitamin-K-Antagonisten Warfarin behandelt wurden. In einer Dosierung von 150 mg zweimal täglich reduzierte der Thrombininhibitor das Risiko für Schlaganfälle oder systemische Embolien um 35\% gegenüber Warfarin bei gleichzeitiger Reduktion der intrakraniellen Blutungen um 59\%.

Auch bei der Sekundärprophylaxe ergab sich in der Subgruppenanalyse laut Grond eine eindeutige Reduktion der Rate an Schlaganfällen und schweren Embolien bei signifikanter Reduktion der Häufigkeit von Hirnparenchymblutungen. Vor dem Hintergrund der hohen Letalität von Hirnblutungen sei dies ein relevanter Befund. Die Daten belegen, dass Dabigatran auch nach transitorischer Attacke (TIA) oder Schlaganfall eine sichere und effektive Option der Antikoagulation darstellt.

- Christine Vetter

Quelle: Symposium „Antikoagulation - die Zukunft hat begonnen", 117. Kongress der DGIM, Wiesbaden, Mai 2011(Veranstalter: Boehringer Ingelheim) 